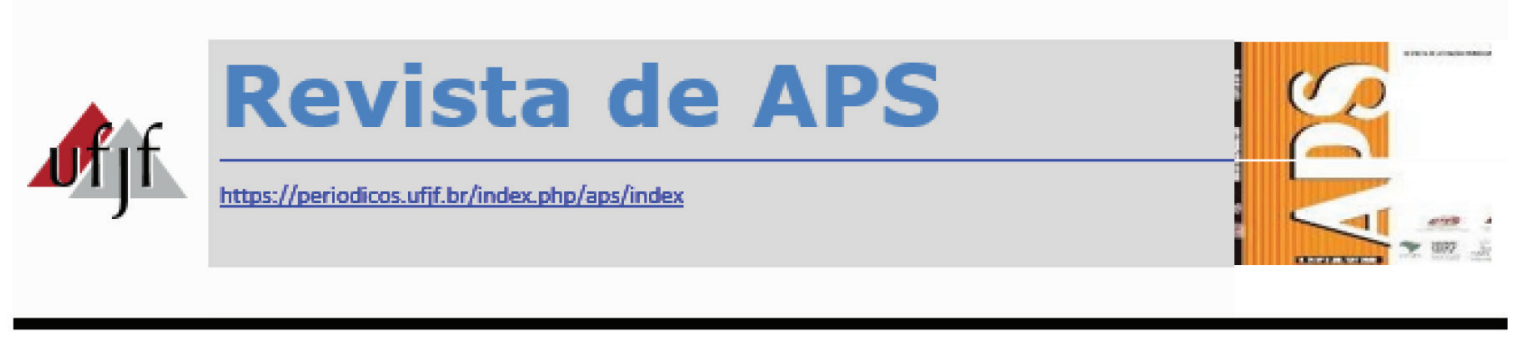

\title{
Tomada de decisão na gerência em Atenção Primária à Saúde: percepção de enfermeiros
}

\section{Decision making in primary health care management: nurses' perception}

\author{
Ingrid Rafaely Alves Saraiva ${ }^{1}$, Ana Paula Gomes Viana², Vinícius Costa Maia \\ Monteiro $^{3}$, Adriano da Costa Belarmino ${ }^{4}$, Jocasta Maria Oliveira Morais ${ }^{5}$, Antonio \\ Rodrigues Ferreira Júnior ${ }^{6}$
}

\begin{abstract}
RESUMO
Objetivo: analisar o processo de tomada de decisão por enfermeiros gerentes na Atenção Primária à Saúde. Método: pesquisa descritiva exploratória, com abordagem qualitativa, realizada com oito enfermeiros de cinco unidades básicas de saúde no município de Mossoró/RN. Os dados foram coletados de abril a julho de 2017, sendo organizados por meio da Análise de Conteúdo. Utilizou-se como base teórica para discussão o Modelo de Tomada de Decisão e Racionalidade Limitada de Herbert Simon. Resultados: A falta de comunicação, estrutura das unidades, sobrecarga de trabalho e o relacionamento interprofissional representam pontos frágeis na execução dos serviços de saúde. Conhecimento teórico-prático gerencial, habilidades cognitivas de liderança e de compreensão na resolução de problemas foram elencados como potencialidades no processo. Conclusão: A tomada de decisão no processo de gerência apresenta fragilidades pungentes que se sobressaem às potencialidades da equipe de saúde, sendo necessário o desenvolvimento de habilidades de cooperação, gestão e assistência integralizados para alcançar qualidade na gerência no nível de atenção primário em saúde.
\end{abstract}

\footnotetext{
${ }^{1}$ Graduanda em Enfermagem - Universidade Potiguar.

${ }^{2}$ Graduanda em Enfermagem - Universidade Potiguar.

${ }^{3}$ Mestrando em Enfermagem - Universidade Federal do Rio Grande do Norte.

${ }^{4}$ Mestrando em Saúde Coletiva - Universidade Estadual do Ceará. E-mail: adrian.belarmino@aluno.uece.br

${ }^{5}$ Mestra em Saúde e Sociedade pelo Programa de Pós-graduação em Saúde e Sociedade da Universidade do Estado do Rio Grande do Norte. Docente da Universidade Potiguar e enfermeira da Estratégia Saúde da Família do município do Assú/RN. Atualmente é enfermeira efetiva do Centro de Atenção Psicossocial - CAPS, do município do Assú/RN.

${ }^{6}$ Doutor em Saúde Coletiva pela Universidade Estadual de Campinas - UNICAMP (2015). Professor da Graduação em Enfermagem e da Pós-graduação em Saúde Coletiva da Universidade Estadual do Ceará. Líder do grupo de pesquisa no diretório do CNPq: Redes Integradas de Saúde. Coordenador atual do Programa de Pós-graduação em Saúde Coletiva da UECE.
} 
PALAVRAS-CHAVE: Gestão em saúde. Profissionais de enfermagem. Atenção Primária à Saúde.

\begin{abstract}
Objective: to analyze the decision-making process by nurse managers in Primary Health Care. Method: descriptive-exploratory research, with a qualitative approach, conducted with eight nurses from five basic health units in the city of Mossoró/RN. The data were collected from April to July 2017 and organized through Content Analysis. The Herbert Simon's Decision-Making and Bounded Rationality Model was used as a theoretical basis for discussion. Results: The lack of communication, structure of the units, work overload and the interprofessional relationship represent fragile points in the execution of health services. Theoretical-practical managerial knowledge, cognitive skills of leadership and understanding in problem solving were listed as potentialities in the process. Conclusion: Decision-making in the management process presents poignant weaknesses that stand out from the health team's potential, requiring the development of integrated cooperation, management and assistance skills to achieve quality management at the level of primary health care.
\end{abstract}

KEYWORDS: Health management. Nursing practitioners. Primary health care.

\title{
INTRODUÇÃO
}

Mundialmente, o modelo de Atenção Primária Mundial é baseado na Declaração de Alma-Ata, que o recomendou como mecanismo-chave para melhoria dos níveis de saúde mundiais, integrando serviços sociais e de saúde. Constitui-se, assim, no elemento central para elevação das capacidades de sustentabilidade e para construção de sistemas de saúde equitativos e acessíveis. ${ }^{1}$ Além disso, engloba ainda atributos de acesso no primeiro contato, abrangência e coordenação dos cuidados, podendo potencializar sua capacidade interativa com a coletividade, caracterizando propriedades de cuidados centralizados na família, orientação comunitária e competência cultural. ${ }^{2}$

No Brasil, a atenção primária configurou-se no ponto central de desenvolvimento das ações de promoção, prevenção, recuperação e reabilitação à saúde, sendo acesso principal ao sistema de saúde brasileiro e constituindo-se no primeiro nível de complexidade na prestação de serviços de saúde e da rede de atenção regionalizada. Entre os resultados da implantação da rede primária de atenção à saúde baseada na Estratégia de Saúde da Família (ESF), destaca-se a diminuição da mortalidade infantil relacionada a causas diversas, aumento da cobertura vacinal e diminuição de internações por causas sensíveis e doenças crônicas. No entanto, cabe destacar que a cobertura populacional é heterogênea nas diversas regiões constituintes do país, com níveis diversificados de implantação e gestão das equipes da estratégia saúde da família. ${ }^{3,4}$ 
Nessa perspectiva, o modelo organizacional de gerência no nível primário de atenção constitui ferramenta fundamental que reproduz as políticas públicas de saúde, traduzindoas em ações concretas efetivadas conjuntamente com a equipe local e a comunidade. Essa dinâmica constituinte configura-se em práticas integradoras e interdisciplinares, objetivando organização dos serviços da atenção primária e resolutividade cristalizada em ações da ESF. ${ }^{5,6}$

Ademais, aborda uma complexa rede de experiências gerenciais e assistenciais, poder decisório e liderança carismática, resolutiva e mediadora de conflitos para mobilização das equipes e criação de ambientes propícios à criatividade, transformação e aplicabilidade no desenvolvimento das ações em saúde. ${ }^{7}$

Nesse contexto, a área de enfermagem, como uma das profissões responsáveis pelo gerenciamento do cuidado e das práticas integrais, configura-se como ator principal nos mecanismos do processo de trabalho em saúde e no desenvolvimento de interfaces de cuidado que compõem e contribuem na construção do Sistema Único de Saúde (SUS). No entanto, é necessário repensar novas opções alternativas que se diferenciem do modelo gerencial hegemônico racionalista. ${ }^{5}$

Contextualmente, a tomada de decisão nos processos gerenciais de saúde consiste em ação humana essencial para desenvolvimento e consolidação do trabalho em equipe. Implica análise de situações e resolução de problemas, ressaltando-se a necessidade de compreensão dos fatores que interferem e influenciam neste processo decisório concernente ao desenvolvimento de atividades de planejamento, comunicação, administração de conflitos, negociação e liderança e como podem comprometer a assistência implementada. ${ }^{8}$

Assim, indaga-se: como ocorre a tomada de decisão de enfermeiros na gerência dentro da atenção primária em saúde?

Ademais, o estudo contribui no campo de gestão em saúde através da efetivação do trabalho em saúde, compreendendo sua organização, processos constitutivos e mecanismos fomentadores com base na subjetividade das ações e atividades na atenção primária em saúde. Além disso, há necessidade de estudos acerca da tomada de decisão e suas implicações nos cuidados primários de saúde e contribui nas problemáticas atuais acerca desse nível de atenção no país.

Há atualmente a necessidade de desenvolvimento de habilidades de tomada de decisão na gestão e na promoção de saúde em atenção primária, sendo uma das prioridades para elevação dos níveis máximos de atenção nos sistemas de saúde mundiais.

Assim, o presente estudo objetiva analisar o processo de tomada de decisão por enfermeiros gerentes na Atenção Primária à Saúde.

\section{MATERIAIS E MÉTODO}

Trata-se de uma pesquisa descritiva, com natureza exploratória e abordagem qualitativa, ${ }^{9}$ realizada em cinco Unidades de Atenção Primária em Saúde (UAPS) de 
Mossoró, segunda cidade com maior população do estado do Rio Grande do Norte, com quase 300.000 habitantes e IDHM de $0,720 .^{10}$

Para o estudo foram selecionados enfermeiros ligados a gerência de UAPS. Para seleção dos participantes utilizou-se a técnica de bola de neve ${ }^{11}$, sendo que 0 entrevistado piloto indicou o segundo e assim por diante, formando a amostra composta por oito enfermeiros atuantes nas UAPS do estudo. Para delimitar o número limite de participantes, utilizamos critérios de saturação teórica, sendo a coleta de dados efetuada no período de abril a julho de 2017.

Foram considerados critérios de inclusão: ser enfermeiro(a) das UAPS selecionadas há pelo menos um ano e atuar na área de gerência. Os critérios de exclusão foram: enfermeiros que estavam de férias, de licença-maternidade ou licença médica. 0 número de participantes resumiu-se a oito enfermeiros.

Foi realizada entrevista individual com os participantes, utilizando como instrumento para coleta dos dados roteiro semiestruturado contendo perguntas sobre informações sociodemográficas e da gerência nas unidades, ${ }^{11}$ aplicada de forma reservada e individual, em sala de livre escolha dos participantes. As entrevistas duraram cerca de 40 minutos, tempo limite em que todos os participantes responderam às perguntas. Foi utilizado para gravação o recurso de gravador de áudio MP3 para registrar os depoimentos, sendo posteriormente transcritas na íntegra. Para manter a identidade dos entrevistados, empregou-se código alfanumérico utilizando a codificação Enf e os números correspondentes. Além disso, para anotar manifestações não verbais corporais e faciais, utilizou-se diário de campo com objetivo de ampliar as informações colhidas.

A pesquisa apontou predominância de sexo feminino (sete), cor branca (seis), com idade entre 43-58 anos, com maioria possuindo especialização em Gestão em Saúde e Saúde da Família (seis), atuação profissional acima de 20 anos (oito), casadas (sete), católicas praticantes (sete), com residência própria (oito) e moradoras da zona urbana (oito).

Os discursos coletados foram organizados por meio da Análise de Conteúdo. ${ }^{12}$ Para discussão dos depoimentos utilizou-se o Modelo de Tomada de Decisão e Racionalidade Limitada de Hebert Simon como ferramenta analítica do processo gerencial, objetivando refletir sobre suas prováveis potencialidades e fragilidades evidenciadas no dia a dia e na construção e produção de resultados baseadas na incerteza, tanto relativamente aos agentes produtores como ao ambiente complexo em que ocorre a tomada de decisão. ${ }^{13}$

Utilizou-se o Consolidated Criteria for Reporting Qualitative Research (COREQ) para ampliar a transparência do processo metodológico da pesquisa. ${ }^{14}$

O projeto foi submetido ao Comitê de Ética em Pesquisa com Parecer no 1.755.704 e CAAE: 59747816.7.0000.5296, no dia 30 de setembro de 2016, respaldado pela Resolução 466/12, ${ }^{15}$ que trata de diretrizes e normas regulamentadoras de pesquisa em seres humanos. 


\section{RESULTADOS}

Adiante, está exposta a situação sociodemográfica dos entrevistados. Posteriormente, as duas categorias com a temática principal Gerência, possibilitando expor os discursos dos depoentes. A figura a seguir esquematiza o processo de construção das categorias temáticas:

Figura 1 - Esquema de construção categorial-temática dos resultados, Mossoró-RN, 2019

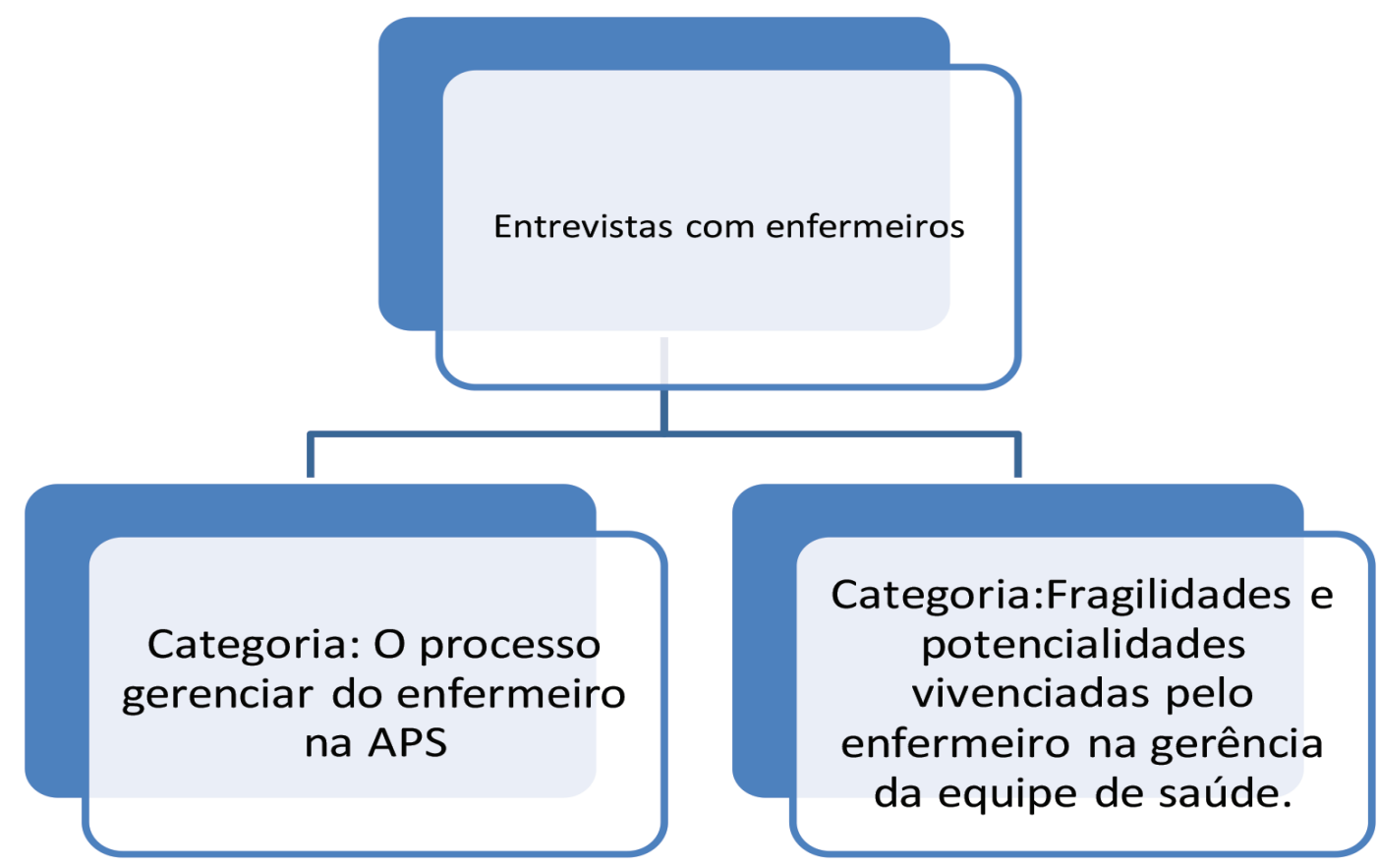

Fonte: Elaborado pelos autores

\section{Categoria: Processo gerenciar do enfermeiro na APS}

Acerca da concepção que o profissional enfermeiro tinha sobre o ato de gerenciar da equipe, os entrevistados trouxeram diversos conceitos:

"Gerenciar é você coordenar uma equipe para que possa fluir os serviços que tanto fique bom para o usuário como para o servidor né? Você tem que ter assim, jogo de cintura, procurar ao máximo possível agradar aos dois lados, tanto para o serviço como para o servidor." (Enf. 005)

"Primeiro, estimular e propiciar um ambiente de equipe; segundo, partilhar atividades, conhecimentos, supervisionar as atividades, acompanhar e também intervir quando for necessário." (Enf. 003)

"Gerenciar é bem amplo né? Por que você vai desde a questão do envolvimento, conhecimento, da sua prática, operacionalização da assistência, do acompanhar, do intervir, de coordenar, supervisionar; então eu vejo de uma forma bem ampla 
mesmo." (Enf. 008)

Ao analisar os discursos, percebe-se que os profissionais possuem respostas variadas acerca da temática, demonstrando saber acerca da função de gerência no desenvolvimento das atividades em saúde, no entanto com limitado domínio acerca do assunto. Apesar de a maioria dos profissionais referirem atuar há mais de 20 anos, a função de gerenciar não é considerada, sendo que ao associá-la com as demais funções e programas propostos pelo Ministério da Saúde, o gerenciamento passa a ser executado na maioria das vezes diante de disponibilidade e tempo.

Refletindo acerca dessa problemática, o profissional enfermeiro apropria-se gradativamente da função gerencial tornando-se o responsável pelo êxito ou fracasso das ações de trabalho em equipe de saúde. Assim, mesmo diante de cenários de desenvolvimento da gestão colegiada, cabe ao enfermeiro conduzir e solucionar problemas da equipe. Alguns entrevistados afirmaram ainda que:

"A gente não gerencia. Tipo assim, a gente tenta organizar, organiza a questão dos programas, senta para montar a estratégia, discutir problemas da área, organiza as palestras, intervenções, essas coisas, a gente tem essa função [...] é tipo, organizar né o serviço [...]. Mas gerenciar, acho que não existe isso, é tanto que existe um gerente aqui." (Enf. 005)

"No ministério a gente não tem esse nome, a gente não entra na equipe como gerente não, pela estratégia, a não ser que mudou alguma coisa; mas assim, eu nunca vi gerente enfermeira da equipe não, a gente organiza, supervisiona, mas não tem essa palavra gerente [...] a gente supervisiona por natureza, pela natureza da enfermagem, o técnico e também nos foi atribuído supervisionar os agentes de saúde [...]." (Enf. 006)

Alguns profissionais mencionaram dificuldade em gerenciar devido à falta de "autonomia" em comandar a equipe de enfermagem. Expõe-se ainda que os profissionais que fazem parte da equipe acabam não tendo o enfermeiro como uma liderança, como gerente de equipe, ou até mesmo supervisor, como foi visualizado em alguns discursos. Nessa perspectiva, sobre as atribuições do enfermeiro enquanto gerente da equipe de enfermagem, relata-se que:

"É muita coisa, porque assim, a gente dá conta do trabalho nosso e além de tudo dá de conta do trabalho dos agentes, dos técnicos de enfermagem principalmente dessas duas categorias, por que dentista a gente já nem vê, e o médico então, está acima de tudo né?" (Enf. 007)

Diante disso, em outros discursos dos entrevistados, foi percebida fragilidade relacionada a exercer a função de gerente da equipe de enfermagem. Por ser uma função que exige liderança, responsabilidade e envolve diretamente as pessoas do campo de trabalho em saúde como também os usuários que utilizam os serviços, muitas vezes relata-se como difícil tarefa, em que existe uma gama de fatores que contribuem para a parcialidade na prática do gerenciamento. Entre os discursos, houve alguns que chamaram maior atenção: 
"É difícil você estar numa função que as vezes você não tem condições de exercer aquilo". (Enf. 007)

"O processo gerenciar da enfermagem na prática, colocar como manda a prática é meio difícil, mas a gente tenta". (Enf. 002)

"[...] Se a gente não conseguir gerenciar nossas atividades na enfermagem como um todo aí o serviço para né? Ou então funciona negativo, a gente vê isso na resposta com usuário [...]". (Enf. 005)

Evidenciou-se através dos discursos que colocar em prática o gerenciamento da equipe de enfermagem tem sido um duelo entre as orientações do Ministério da Saúde e o que é efetivado na prática, pois gerenciar além dos diversos requisitos relacionados à competência interpessoal, flexibilidade, espírito inovador e criatividade, também envolve recursos materiais para executar os planos e metas da equipe. A falta de investimentos na atenção básica em saúde afeta principalmente a gerência da equipe e os planos e metas estipuladas para a promoção e prevenção em saúde para a população.

A falta de condições de trabalho foi mencionada em todas as entrevistas concedidas pelos profissionais enfermeiros quando questionados se o gerenciamento de enfermagem era realmente colocado em prática, destacando-se nos seguintes depoimentos:

"A gente tenta né, com muitas falhas, mas a gente tenta cumprir algumas coisas e aí como a gente já relatou de acordo com os cuidados, a falta de condições de trabalho, então a gente acaba fragilizando [...]". (Enf. 008)

"É, porque a gente tem que fazer a prática de qualquer maneira né? Pode não ser $100 \%$, mas a gente tem que fazer". (Enf. 007)

Diante disso, existiram profissionais que se mostraram extremamente angustiados em relação ao gerenciamento da equipe de enfermagem. Tendo em vista o discurso da Enf. 8, que deixou clara a dificuldade em comandar uma equipe, o gerenciamento da unidade de saúde diversas vezes está relacionado a pessoas de múltiplas áreas de saberes.

\section{Categoria: Fragilidades e potencialidades vivenciadas pelo enfermeiro na gerência da equipe de saúde}

Algumas falas de profissionais relataram os problemas vivenciados no dia a dia quando foi perguntado sobre quais as dificuldades encontradas no gerenciamento de enfermagem:

"As dificuldades que tem no gerenciamento hoje é essa parte de recursos humanos que está muito difícil, as equipes geralmente são desfalcadas, médico é difícil, vem pouco, tem atendente pouco, falta condições; as vezes você faz um atendimento e não tem o retorno adequado, subdimensioamento da equipe e também falta de recursos estruturais, insumos, esses tipos de coisas. Você 
pede um conserto, você pede um profissional e não tem retorno, não volta. Você acaba trabalhando com o que tem". (Enf. 002)

"Acho que é essa sobrecarga né? Hoje todo mundo tem mais de um vínculo, os agentes de saúde, os técnicos de enfermagem, os enfermeiros, por que antigamente era só os médicos, mas hoje não, todos têm; então assim a gente tem uma carga horário muito pesada, e por conta desses desencontros, do cumprimento da carga horária também, todos os profissionais dão suas 40 horas; além de recursos que falta, de condições de trabalho [...] Isso são coisas que impedem o melhor gerenciamento." (Enf. 008).

Em todos relatos dos enfermeiros entrevistados foram visualizadas dificuldades em gerenciar a equipe de enfermagem. Diante disso, percebem-se vários fatores que dificultam a efetivação da gerência: o "poder" entre a gerência da unidade, a falta de profissionais para suprir as demandas da unidade, o comprometimento com o serviço.

Em quase todas as entrevistas, foi percebido que, entre os principais problemas encontrados diante o gerenciamento, destaca-se a falta de recursos humanos e a desvalorização profissional, as quais acabam agregando diversos outros fatores para contribuir na parcialidade da execução do gerenciamento.

Acerca do questionamento da carga horária adequada para desenvolver o gerenciamento em saúde, alguns entrevistados trouxeram que:

"A carga horária que a gente cumpre é suficiente, o problema são as outras engrenagens, certo? Aí acaba de certa forma desmotivando, mas no geral dá certo." (Enf. 002)

"Se é suficiente eu não sei, mas a gente já dá 40 horas né? Então não tem como expandir mais do que isso não. Já é de segunda a sexta de manhã e de tarde, então, tem que ser suficiente. Agora não é tão suficiente porque a gente tem muita atribuição que não era para ser nossa, que deveria ser dividido entendeu? Se cada um fizesse sua parte facilitava muito, dava tempo sobrando." (Enf. 007)

Relacionando ao gerenciamento e diante das diversas dificuldades existentes, os profissionais entrevistados avaliaram a questão da sobrecarga de trabalho. Alguns profissionais disseram que:

"A sobrecarga existe porque a gente não faz só o processo gerenciar a gente faz todos os processos dentro da unidade. Aí além disso tudo tem o atendimento, tem os programas, tem as visitas tem a supervisão do ACS [...]" (Enf. 002)

"Eu acho assim, existe uma sobrecarga de trabalho não enquanto gerente da equipe de enfermagem, mas sim enquanto função enfermeira. A gente vai absorvendo coisas que não é da função da gente. Muitas vezes a gente tá fazendo por exemplo, um pré-natal e alguém bate na porta pedindo uma declaração de residência, outra bate atrás de uma receita, e por aí vai [...]" (Enf. 006)

"Já respondi. Existe! Em cima de um enfermeiro existe! Eu creio, eu rezo que esses próximos profissionais, médicos principalmente, venham com uma visão diferente sobre saúde pública, queiram realmente fazer saúde pública, além da saúde curativa, lógico, mas que eles venham com uma visão diferente, porque esses mais antigos a visão é muito curativa". (Enf. 007) 
Quando questionados acerca do gerenciamento deficiente da equipe de enfermagem como aspecto que atrapalha nas atividades do cotidiano dos serviços, alguns profissionais disseram:

"Quando não existe o entendimento né da equipe toda, entre a parte dos enfermeiros, os técnicos, não tiver um bom entendimento realmente o serviço não anda né, não flui normalmente". (Enf. 005)

"Atrapalha muito, porque na hora que você não gerencia, não organiza, você perde até o contato com o serviço, com a equipe, com a organização do serviço, e isso com certeza vai comprometer a assistência". (Enf. 003)

Em todas as falas transcritas até aqui, são descritas dificuldades em gerenciar a equipe de enfermagem. Vários são os motivos relatados, destacando-se: os conflitos da equipe de enfermagem, falta de autonomia, responsabilidade, carga horária e sobrecarga de trabalho. Apesar de todas as dificuldades, acredita-se que na prática do gerenciamento também existam potencialidades. Os colaboradores da pesquisa relataram sobre tal assunto:

"A questão de humildade né, de saber coordenar de saber compreender, está sempre junto com a equipe, trabalhar junto, lado a lado". (Enf. 005)

"Acho que para potencializar tem que ver a questão assim, de entrosamento, tem que se esforçar muito mesmo por que as dificuldades são maiores que os facilitadores né, por que tem o subdimensionamento de pessoas". (Enf. 002)

"Sim. Disponibilidade, você está disposto a trabalhar o gerenciamento, por ser um elemento primordial, outro elemento é o conhecimento, outro elemento seria a facilidade de lidar com o outro que também eu acho que isso é importante". (Enf. 003)

As competências gerenciais são pouco exploradas nos discursos expostos, mas ressaltam-se habilidades do enfermeiro diante dos processos em gerência e múltiplas fragilidades no processo de gerência.

\section{DISCUSSÃO}

Os discursos analisados contextualmente evidenciam as fragilidades inerentes ao processo de gerência no nível de atenção básica em saúde na região Nordeste do Brasil. A APS constitui o primeiro nível de cuidado procurado pelas pessoas, sendo constituída por equipes de Saúde da Família performando uma equipe de saúde composta por médico de família, enfermeiro, técnicos de enfermagem, agentes comunitários de saúde e especialistas em outras áreas de saúde. ${ }^{16}$

Assim como em outros locais do mundo, no Brasil ocorre nos últimos anos a expansão da APS para Cobertura Universal de Saúde, objetivo estimulado pelas Nações Unidas, Organização Mundial da Saúde e outras organizações de saúde 
globalmente, para estabelecer e propiciar modelos assistenciais, sistemas de saúde efetivos e capacitar profissionais atuantes em atenção primária. Nessa perspectiva, maximizar as habilidades avançadas da enfermagem e sua capacidade em cuidados primários em saúde são apontados por evidências como mecanismo-chave para atingir esse objetivo. ${ }^{17}$

Acerca dos depoimentos, percebe-se a fragilidade em organizar e sistematizar ações gerenciais, organizacionais, relacionadas à linha de cuidado e aos recursos humanos na área das comunidades atendidas na região das UBS, delimitando as habilidades relativas ao enfermeiro como tomador de decisão de território de vida dos usuários do sistema de saúde.

Na América Latina, países como Brasil, Colômbia, México e Chile, através de planos estratégicos desde 2014 da Organização Pan-Americana da Saúde (OPAS), vêm investindo na Cobertura Universal focada em Atenção Primária e no desenvolvimento de recursos humanos avançados, principalmente de enfermeiros de práticas avançadas (EPA). Caracteristicamente, têm por base conhecimento especializado, habilidades complexas de tomada de decisões e competências clínicas na prática, sendo que países como Estados Unidos, Canadá, Holanda e Austrália vêm desenvolvendo e implementando as EPAs há 50 anos. ${ }^{18}$

O Chile foi além nas orientações da OPAS, desenvolvendo um sistema de cobertura abrangente e fortalecendo a APS no país na última década. Denominado de Modelo Abrangente de Saúde da Família e Comunidade, compreende a modificação do modelo paternalista de atenção ao usuário para uma abordagem participativa, com atuação dos usuários nos processos de gestão da saúde, envolvimento comunitário nos processos consultivos e avaliativos em saúde. ${ }^{18,19}$

A complexidade da APS exige transformações nos processos de trabalho, principalmente relacionadas às novas realidades da globalização e das imposições dessa dinâmica à sociedade e saúde. Assim, exigem-se novas habilidades, conhecimentos e atitudes, que as Diretrizes Curriculares Nacionais, do Ministério da Educação, traduzem em seis competências: atenção à saúde; tomada de decisão; comunicação; liderança; administração e gerenciamento; educação permanente. ${ }^{20}$

Nessa perspectiva, a tomada de decisão em saúde implica a limitação humana tanto física quanto intelectual, relativa à complexidade em processar todas as informações necessárias, dominar o tempo, resolver as situações e minimizar as incertezas nas organizações. ${ }^{21}$

Nesta perspectiva, enfoca-se no estudo o Modelo de Tomada de Decisão e Racionalidade Limitada de Simon ${ }^{21}$, descrito na figura abaixo: 
Figura 2 - Adaptação do Modelo de Tomada de Decisão e Racionalidade Limitada, 2019

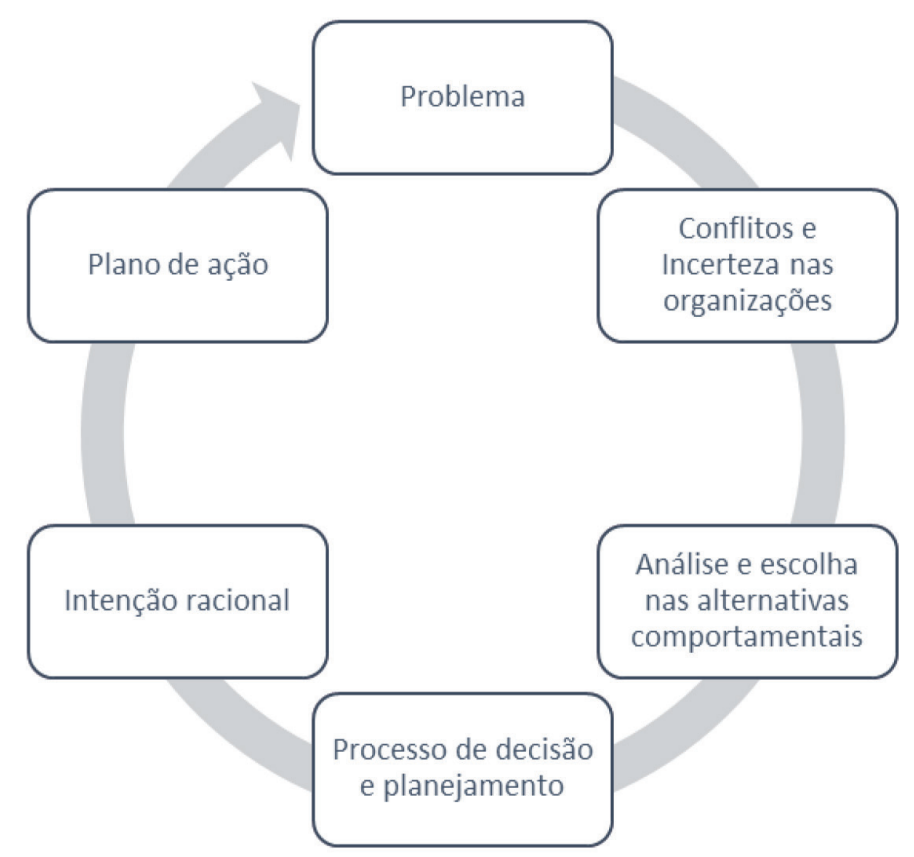

Fonte: Pierangeli; Machado; Sales, 2017

Acerca do Modelo de Tomada de Decisão, Hebert Simon define o processo decisório como curso de pensamento e ação que resultará em uma seleção. Ele se baseia no julgamento para análise e ação no processo de tomada de decisão, sendo que esses elementos são instrumentos para definir o problema, avaliar opções e atingir a decisão. Nessa dinâmica, a racionalidade possui destaque pois implica ao tomador de decisão adequar-se ao sistema integrado, utilizar-se de alternativas disponíveis, da análise das prováveis consequências da escolha e criteriosamente definir a opção. ${ }^{22}$

Nos discursos dos entrevistados observa-se que as decisões em saúde são complexas e o despreparo implica na limitação gerencial. Nesse contexto, adotando-se o modelo decisório, três etapas principais destacam-se: identificação do momento para tomada de decisão, análise situacional e escolha da alternativa melhor, ${ }^{8}$ no entanto não se delimita nenhum processo organizacional na realização das atividades gerenciais citadas.

Nessa dinâmica, a racionalidade limitada utilizada no modelo de Simon implica na incerteza na previsão de eventos futuros e na incapacidade de obtenção e processamento de todas as informações necessárias para a tomada de decisão, relacionados à complexidade do sistema. ${ }^{23}$

Um dos principais obstáculos apontados na implementação de cuidados de saúde no Brasil na APS consiste nas dificuldades socioeconômicas, desigualdades regionais e socioculturais, que aliadas a diminutos recursos humanos de profissionais de saúde e médicos especializados na área e dificuldades na coordenação da atenção entre setores 
de APS e de atenção especializada, determinam comprometimentos nas atividades do enfermeiro da ESF. ${ }^{24}$

Os depoentes referem em diversos depoimentos o déficit na disponibilidade de profissionais, principalmente médicos, e a consequente sobrecarga de trabalho oriunda das diversas funções desempenhadas, sendo que se confunde nos depoimentos a gerência da assistência, de recursos humanos e organizacional.

Assim, o conceito de gestão/administração refere-se ao conhecimento aplicado no manejo de organizações de saúde relacionado às ações de gerenciar redes de atenção e unidades de assistência tanto de esferas públicas como privadas. Envolve inúmeras dimensões como gestão de pessoas, relacionada à gestão de relações de trabalho e da força de trabalho em número e qualificação; gestão de materiais e processos, referente a estrutura, fluxos organizacionais, processos de trabalho, materiais e equipamentos; e gestão financeira. ${ }^{25}$

Além da sobrecarga de trabalho e carga horária, a implementação das atividades e do cuidado foi destacada como fragilidade durante o desenvolvimento da pesquisa. Relativo a isso, em estudo efetuado acerca do dimensionamento de enfermagem e do processo de trabalho em uma UBS do Centro-Oeste de São Paulo, evidenciou-se falta de recursos humanos, implicações para o processo de trabalho relacionado a monitoramento do sistema de informação e reorganização da abrangência do serviço de saúde. ${ }^{26}$

A racionalidade na tomada de decisão abrange obtenção de informações que maximizem as escolhas, possibilitem a análise das alternativas com menor nível de incerteza e possibilitem planejamento e implementação da alternativa escolhida, no entanto abrange as limitações relacionadas à racionalidade. ${ }^{8}$ As incertezas diante dos processos nas unidades básicas de saúde pesquisadas elencam, entre outras, a falta de comunicação entre a equipe multiprofissional, déficit na organização das atividades gerenciais e nas habilidades dos enfermeiros relacionado ao processo decisório, que foram evidentes.

O conceito de racionalidade limitada abrange tanto limites relativos ao tomador de decisão como o ambiente e envolve três tipos: limites cognitivos de indivíduos e organizações, que apresentam capacidade limitada para coletar, processar e interpretar informações; a complexidade do processo decisório, em que relações causais e regularidades são indiscerníveis no ambiente em que o agente atua; e a incerteza relativa a eventos exógenos. ${ }^{27}$

O processo decisório em saúde é complexo e imprevisível, envolvendo decisões tanto programadas como não programadas, em que a certeza se caracteriza pela situação em que toda informação necessária para tomada de decisão encontrase disponível, principalmente no nível operacional. ${ }^{28}$ Nas entrevistas efetuadas observa-se incerteza nas ações executadas inclusive no nível operacional de gerência 
das atividades de APS.

Assim, não há nos discursos evidências de processos de gerência e planejamento das atividades em saúde, sendo a competência de tomada de decisão desconhecida e o processo desconhecido, não sendo implementado.

Acerca disso, há necessidade de desenvolver mecanismos potencializadores no gerenciamento das equipes de saúde, principalmente relativos à tomada de decisão nesse âmbito. Capacitações, oficinas de liderança, rodas de conversa são meios de promover habilidades do enfermeiro, além de maximizar sua atuação no aperfeiçoamento da equipe, da unidade e, consequentemente, na prestação de serviços de saúde de qualidade para a população adscrita.

\section{CONCLUSÕES}

A tomada de decisão no processo de gerência na atenção primária apresenta fragilidades pungentes que se sobressaem às potencialidades da equipe de saúde, dificultando a atuação desta em enfermagem no desenvolvimento de suas competências. Acerca de elementos fortalecedores da gerência de unidades primárias de saúde, o processo decisório compartilhado com a equipe interprofissional de saúde e princípios de liderança e cooperação mútua são importantes processos a serem considerados.

Relativamente a limitações, a principal relaciona-se ao acesso a enfermeiros de diferentes unidades para performar um perfil do município, pois a técnica de determinação dos participantes não permite obter segurança nesse quesito. No entanto, as similaridades com espaços de atenção primária no Brasil e no mundo tornam replicáveis seus resultados.

É necessário o desenvolvimento de elementos fortalecedores na perspectiva da gerência de saúde pelos enfermeiros de UAPS para melhoria de habilidade de cooperação, gestão e assistência de modo integralizado, objetivando alcançar qualidade na gerência no nível de atenção primário em saúde.

\section{REFERÊNCIAS}

1. Tao W, Zeng W, Yan L, Yang H, Wen J, Li W. The health service capacity of primary health care in West China: different perspectives of physicians and their patients.

I. BMC Health Services Research. 2019;19:143. Disponível em: https://doi. org/10.1186/s12913-019-3964-x3.

2. Rolim LB, Monteiro JG, Meyer APGFV, Nuto SAS, Araújo MFM, Freitas RWJF. Evaluation of Primary Health Care attributes of Fortaleza city, Ceará State, Brazil. Rev Bras Enferm. 2019;72(1):19-26. Disponível em: http://dx.doi.org/10.1590/0034- 
7167-2018-0033.

3. Shimizu HE, Ramos MC. Evaluation of quality of the family health strategy in the Federal District. Rev Bras Enferm. 2019;72 (2):367-74. Disponível em: http://dx.doi. org/10.1590/0034-7167-2018-0130.

4. Barreto ACO, Rebouças CBA,Aguiar MIF, Barbosa RB, Rocha SR, Cordeiro, LM, et al. Perception of the Primary Care multiprofessional team on health education. Rev. Bras. Enferm. 2019; 72 (Suppl 1):266-73. [Thematic Issue: Work and Management in Nursing]. Disponível em: http://dx.doi.org/10.1590/0034-7167-2017-0702.

5. Silva SS, Assis MMA, Santos AM. Enfermeira como protagonista do gerenciamento do cuidado na estratégia saúde da família: diferentes olhares analisadores. Texto Contexto Enferm. 2017; 26(3):e1090016. Disponível em: https://doi. org/10.1590/0104-07072017001090016.

6. Nunes LO, Castanheira ERL, Dias A, Zarili TFT, Sanine RR, Mendonça CS, et al. Importância do gerenciamento local para uma atenção primária à saúde nos moldes de Alma-Ata. Rev Panam Salud Publica. 2018; 42:e 175. Disponível em: https://doi. org/10.26633/RPSP.2018.175.

7. Penedo RM, Gonçalo CS, Quelus DP. Gestão compartilhada: percepções de profissionais no contexto de Saúde da Família. Interface (Botucatu). 2019; 23:e170451. Disponível em: https://doi.org/10.1590/Interface.170451.

8. Eduardo EA, Peres AM, Almeida ML, Roglio KD, Bernardino E. Analysis of the decision-making process of nurse managers: a collective reflection. Rev Bras Enferm. 2015; 68(4):668-75. Disponível em: http://dx.doi.org/10.1590/0034$7167.2015680414 \mathrm{i}$.

9- Lakatos EM. Metodologia do trabalho científico. 7ạ ed. São Paulo: Atlas; 2010.

10. Brasil. Instituto Brasileiro de Geografia e Estatística [Internet]. [acesso em 2019 dez 6]. Disponível em: https://cidades.ibge.gov.br/brasil/rn/mossoro/panorama.

11. Freitas ICF, Silva CN, Adan LFF, Kitaoka EG, Paolilo RB, Vieira LA. Pesquisa qualitativa em saúde: um olhar inovador sobre a produção do conhecimento científico. Rev. Baiana de Saúde Pública. 2011 out-dez; 35(4):1001-12. Disponível em: http:// docplayer.com.br/3397592-Artigo-de-revisao-pesquisa-qualitativa-em-saude-umolhar-inovador-sobre-a-producao-do-conhecimento-cientifico.html.

12- Bardin L. Análise de conteúdo. 4ạ ed. 2010.

13. Melo TM, Fucijdi JR. Racionalidade limitada e a tomada de decisão em sistemas complexos. Rev Econ Polit. 2016; 36 (3):622-45. Disponível em: https://doi. org/10.1590/0101-31572016v36n03a09.

14. Tong A, Sainsbnury $P$, Criag J. Consolidated criteria for reporting qualitative research (COREQ): a 32 item checklist for interviews and focus groups. International J Qual Health 
Care. 2007; 19(6):349-57. Disponível em: https://doi.org/10.1093/intqhc/mzm042.

15. Ministério da Saúde do Brasil. Conselho Nacional de Saúde. Resolução no 466, de 12 de dezembro de 2012. Diretrizes e normas regulamentadoras de pesquisas envolvendo seres humanos. Diário Oficial da União da República Federativa do Brasil. 2013 jun [citado em 2017 abr 25]; 150(112 Seção 1):59-62. Disponível em: http://conselho.saude.gov.br/resolucoes/2012/Reso466.pdf.

16. Corrêa DSRC, Moura AGOM, Quito MV, Souza HM, Versiani LM, Leuzzi S, et al. Movimentos de reforma do sistema de saúde do Distrito Federal: a conversão do modelo assistencial da Atenção Primária à Saúde. Ciênc. Saúde Colet. 2019; 24(6):2031-41. Disponível em: http://doi.org./10.1590/141381232018246.08802019.

17. Honig J, Lindrud SD, Dohrn J. Moving towards universal health coverage: advanced practice nurse competencies. Rev. Latino-Am. Enfermagem. 2019; 27:e3132. Disponível em: http://dx.doi.org/10.1590/1518-8345.2901.313.

18. Aguirre-Boza F, Mackay MC, Pulcini J, Bryant-Lukosius D. Estratégia de implementação para a prática avançada de enfermagem na Atenção Primária à Saúde no Chile. Acta Paul Enferm. 2019; 32(2):120-8. Disponível em: https://doi. org/10.1590/1982-0194201900018.

19. Bravo P, Dois A, Contreras A, Soto G, Mora I. Participación de los usuarios en las decisiones clínicas en la atención primaria de salud en Chile. Rev Panam de Salud Publica. 2018; 42:e133. Disponível em: https://doi.org/10.26633/RPSP.2018.133.

20. Siqueira CL, Bernadeli ACF, Gasparino RC, Feldman LB, Cunha ICKO, Oliveira RA. Knowledge of responsible technical nurses on management skills: a qualitative study. Rev Bras Enferm. 2019; 72(1):43-8. Disponível em: http://dx.doi. org/10.1590/0034-7167-2017-0761.

21. Pierangeli MV, Machado LK, Sales RS. Metodologia de Gestão de Riscos aplicada no apoio à Tomada de Decisão: Abordagem de aspectos da Racionalidade Limitada. Revista Espacios. 2017; 38(14):23. Disponível em: https://www.revistaespacios.com/ a17v38n14/a17v38n14p23.pdf.

22. Pacheco Júnior JMC, Gomes R. Tomada de decisão e alta administração: a implantação de projetos de mudanças de gestão da clínica em hospitais do SUS. Ciênc. Saúde Colet. 2016;21(8):2485-95. Disponível em: http://doi. org./10.1590/1413-81232015218.20012015.

23. Melo TM, Fucidji JR. Racionalidade limitada e a tomada de decisão em sistemas complexos. Revista de Economia Política. 2016 jul-set; 3 (3):622:45. Disponível em: https://doi.org/10.1590/0101-31572016v36n03a09.

24. Oliveira AR, Sousa YG, Diniz IVA, Medeiros SM, Martiniano C, Alves M. The daily routine of nurses in rural areas in the Family Health Strategy. Rev Bras Enferm. 2019; 72(4):918-25. Disponível: http://dx.doi.org/10.1590/0034-7167-2018-0243. 
25. Pires DEP, Vandresen L, Machado F, Machado RR, Amadigi FR. Gestão em saúde na atenção primária: o que é tratado na literatura. Texto Contexto Enferm. 2019; 28:e20160426. Disponível em: http://dx.doi.org/10.1590/1980-265XTCE-2016-0426.

26. Santos LC, Andrade J, Spiri WC. Dimensionamento de profissionais de enfermagem: implicações para o processo de trabalho na estratégia saúde da família. Esc Anna Nery. 2019; 23(3): e20180348. Disponível em: http://doi.org./10.1590/2177-9465EAN-2018-0348.

27. Pondé JL. Racionalidade, incomensurabilidade e história: um diálogo entre as obras de Herbert Simon e Thomas Kuhn. Nova Econ. 2017; 27(3):443-76. Disponível em: https://doi.org/10.1590/0103-6351/2836.

28. Nunes NF, Silva AV. Modelos, métodos e teorias utilizados no estudo da decisão e suas aplicações. Humanidades \& tecnologia em revista (FINOM). 2019 jan-dez; 13(16). Disponível em: http://revistas.icesp.br/index.php/FINOM_Humanidade_ Tecnologia/article/view/676. 